\title{
New methods for B decay constants and form factors from Lat- tice NRQCD
}

\author{
Christine Davies $^{1, \star}$, Ciaran Hughes ${ }^{2,3}$, and Christopher Monahan ${ }^{4,5}$ \\ ${ }^{1}$ SUPA, School of Physics and Astronomy, University of Glasgow, Glasgow, G12 8QQ, UK \\ ${ }^{2}$ Fermi National Accelerator Laboratory, Batavia, Illinois 60510, USA \\ ${ }^{3}$ Department of Applied Mathematics and Theoretical Physics, University of Cambridge, Cambridge, CB3 \\ OWA, UK \\ ${ }^{4}$ Institute for Nuclear Theory, University of Washington, Seattle, Washington 98195, USA \\ ${ }^{5}$ New High Energy Theory Center and Department of Physics and Astronomy, Rutgers, the State University \\ of New Jersey, 136 Frelinghuysen Road, Piscataway, New Jersey 08854, USA
}

\begin{abstract}
We determine the normalisation of scalar and pseudo scalar current operators made from NonRelativistic QCD (NRQCD) $b$ quarks and Highly Improved Staggered (HISQ) light quarks through $O\left(\alpha_{s} \Lambda_{\mathrm{QCD}} / m_{b}\right)$. We use matrix elements of these operators to extract $B$ meson decay constants and form factors and compare to those obtained using the standard vector and axial vector operators. We work on MILC second-generation $2+1+1$ gluon field configurations, including those with physical light quarks in the sea. This provides a test of systematic uncertainties in these calculations and we find agreement between the results to the $2 \%$ level of uncertainty previously quoted.
\end{abstract}

\section{Introduction}

The accuracy of the determination of weak decay matrix elements from lattice QCD is critical to the flavour physics programme of over-determination of the Cabibbo-Kobayashi-Maskawa (CKM) matrix in the search for new physics. As well as improving the accuracy where possible, it is important to test the systematic uncertainties by doing the calculations in multiple different ways. This includes using different formalisms for the quarks and also using different methods for the same formalisms.

Here we study matrix elements for $B$ mesons obtained using the NRQCD formalism for $b$ quarks and the HISQ formalism for light quarks. State-of-the-art calculations were carried out in [1] for the $B$ decay constant using an NRQCD Hamiltonian [2] and a temporal axial current operator both improved through $\alpha_{s} \Lambda_{\mathrm{QCD}} / m_{b}$ to obtain a $2 \%$ overall uncertainty. This uncertainty was dominated by the perturbative matching of the nonrelativistic current to that in the continuum, which was done using perturbation theory through $O\left(\alpha_{s}\right)$.

Here we repeat this calculation, but using a pseudoscalar current matched to the same level of accuracy. This method has different systematic uncertainties to those of the original method. Hence the comparison provides a test of our error budget.

In a similar way, the systematic uncertainties in the semileptonic form factor for $B \rightarrow \pi \ell v$ from the traditional method of using a vector current can be tested by employing a scalar current.

${ }^{\star}$ Speaker, e-mail: christine.davies@glasgow.ac.uk 


\section{Normalisation of Lattice NRQCD current operators}

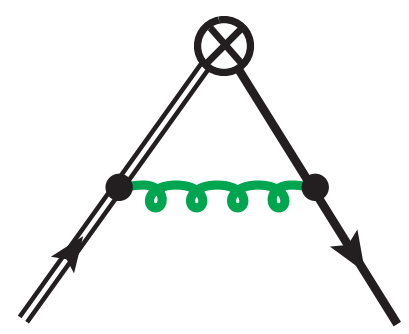

Figure 1. Feynman diagram for the vertex renormalisation in continuum QCD perturbation theory. The heavy quark is denoted by a double line, the light quark by a single line and the exchange of a gluon by a curly line. The current is denoted by a cross inside a circle.

The hadronic parameters, decay constants and form factors, that are needed to determine meson weak decay rates are defined as the matrix elements of current operators appropriate to a continuum formulation of QCD. By expressing the continuum current operator in terms of lattice QCD current operators we can determine these matrix elements in a lattice QCD calculation.

Here we will consider matrix elements for $B$ mesons and make use of the fact that the $b$ quark is nonrelativistic inside the $B$ meson. On the lattice we then use a non-relativistic formulation, lattice NRQCD, for the $b$ quark and combine this with the Highly Improved Staggered Quark (HISQ) formulation for the $u, d$, or $s$ quark inside the $B_{(s)}$ meson.

The continuum heavy-light current that couples to a $W$ boson can be systematically expressed as a non-relativistic expansion in terms of lattice NRQCD-HISQ current operators multiplied by coefficients which can be calculated in perturbation theory as a power series in $\alpha_{s}$. The coefficients depend on the bare NRQCD quark mass and are calculated by matching a heavy quark to light quark scattering process induced by the continuum current and by the combination of NRQCD-HISQ currents. A key Feynman diagram is the one shown in Fig. 1. In [1] (based on perturbative calculations from [3]) the following expansion of the continuum temporal axial current in terms of lattice NRQCD current operators was used

$$
A_{0}=\left(1+\alpha_{s} z_{0}^{A_{0}}\right) \times\left(J_{A_{0}, l a t}^{(0)}+\left(1+\alpha_{s} z_{1}^{A_{0}}\right) J_{A_{0}, l a t}^{(1)}+\alpha_{s} z_{2}^{A_{0}} J_{A_{0}, l a t}^{(2)}\right)
$$

Here

$$
\begin{aligned}
& J_{A_{0}, l a t}^{(0)}=\bar{q}(x) \gamma_{5} \gamma_{0} Q(x) \\
& J_{A_{0}, l a t}^{(1)}=-\frac{1}{2 m_{b}} \bar{q}(x) \gamma_{5} \gamma_{0} \gamma \cdot \nabla Q(x) \\
& J_{A_{0}, l a t}^{(2)}=-\frac{1}{2 m_{b}} \bar{q}(x) \gamma \cdot \nabla \gamma_{5} \gamma_{0} Q(x)
\end{aligned}
$$

where $q$ is a light quark field and $Q$ a heavy quark field and the two derivatives act in opposite directions in $J^{(1)}$ and $J^{(2)}$. Eq. 1 represents an expansion of the continuum current accurate through $O\left(\alpha_{s} \Lambda_{\mathrm{QCD}} / m_{b}\right)$ since all currents that can appear at this order are included. The behaviour of $z_{0}^{A_{0}}, z_{1}^{A_{0}}$ and $z_{2}^{A_{0}}$ as a function of the lattice NRQCD mass in lattice units, $a m_{b}$, is shown in Figure 2. All the $z_{i}$ are well-behaved over the region of $a m_{b}$ values in which we work (appropriate for lattice spacings between $0.15 \mathrm{fm}$ and $0.09 \mathrm{fm}$ ).

By determining the matrix elements of these three currents between the vacuum and a $B$ meson in a lattice QCD calculation, [1] was able to determine the $B$ meson decay constant using:

$$
\left\langle 0\left|A_{0}\right| B(\vec{p}=0)\right\rangle=f_{B} M_{B}
$$




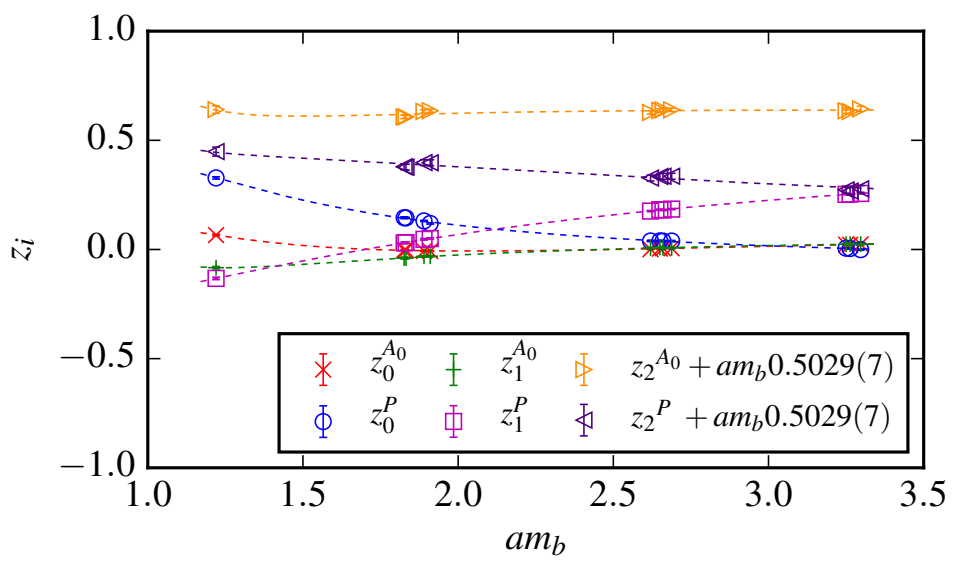

Figure 2. $z_{0}$ and $z_{1}$ factors for the $O\left(\alpha_{s}\right)$ matching of the temporal axial and pseudoscalar NRQCD-HISQ currents to continuum QCD (eqs (1) and (5)) plotted against the bare heavy (b) quark mass in the lattice NRQCD Lagrangian. The part of $z_{2}$ which is linear in $a m_{b}$ has been removed for clarity, but is given in the key.

Here we use the alternative continuum expression:

$$
m_{b}\left(1+\frac{m_{l}}{m_{b}}\right)\langle 0|P| B(\vec{p}=0)\rangle=f_{B} M_{B}^{2}
$$

The continuum pseudoscalar density, $P$, can also be expressed as an expansion in terms of NRQCDHISQ currents. Because $\gamma_{0} Q=Q$, the contributing NRQCD-HISQ currents are in fact the same ones that appear in the expansion of $A_{0}$. We find

$$
m_{b}^{\overline{M S}} P=m_{b}\left(1+\alpha_{s} z_{0}^{P}\right) \times\left(J_{A_{0}, l a t}^{(0)}-\left(1+\alpha_{s} z_{1}^{P}\right) J_{A_{0}, l a t}^{(1)}+\alpha_{s} z_{2}^{P} J_{A_{0}, l a t}^{(2)}\right)
$$

through $O\left(\alpha_{s} \Lambda_{\mathrm{QCD}} / m_{b}\right)$. The $z$ coefficients are related to those for $A_{0}$. Figure 2 shows the different $z$ coefficients as a function of the lattice NRQCD $b$ quark mass. Similar behaviour is seen for $z_{i}^{P}$ as for $z_{i}^{A_{0}}$. Exactly the same coefficients pertain to the scalar operator because of the chiral symmetry of the HISQ action.

\section{Results}

To determine the $B$ and $B_{s}$ meson decay constants the authors of [1] calculated the appropriate matrix elements for NRQCD-HISQ current operators (given in eq. 2) on eight different ensembles of gluon fields generated by the MILC collaboration [4]. These include $u, d, s$ and $c$ quarks in the sea with the masses of the $u$ and $d$ quarks taken to be the same. The ensembles chosen cover three values of the lattice spacing, approximately $a=0.15 \mathrm{fm}, 0.12 \mathrm{fm}$ and $0.09 \mathrm{fm}$. Multiple values of the $u / d$ sea quark mass are also explored from approximately one-fifth of the $s$ quark mass down to the physical value $\left(m_{s} / 27.4\right.$ [5]). NRQCD $b$ quark propagators calculated on these gluon fields are combined with either a valence light quark propagator (with the same mass as the $u / d$ quarks in the sea) or a valence $s$ quark propagator with a tuned mass which typically differs slightly from the $s$ mass in the sea [6]. 


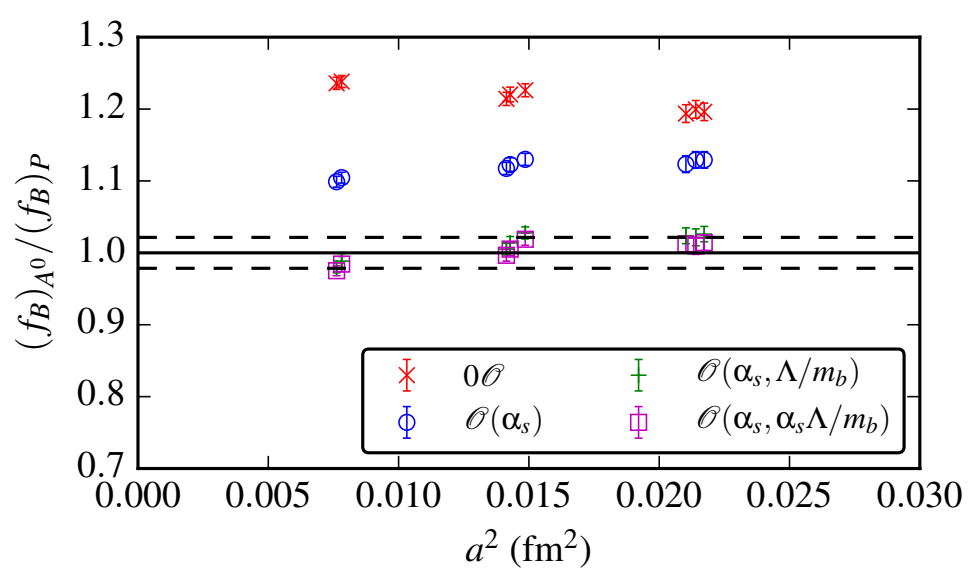

Figure 3. The ratio of the $B$ meson decay constant obtained using the temporal axial current to that obtained using the pseudoscalar density, plotted against the square of the lattice spacing. Results are obtained using lattice QCD calculations for NRQCD $b$ quarks and HISQ light quarks and plotted for successively more accurate non-relativistic expansions of the continuum current operator. We use a variety of ensembles with different lattice spacing and sea quark masses (giving multiple results at each value of the lattice spacing). Red crosses denote the lowest (zeroth) order in the nonrelativistic expansion, while blue open circles include only $J_{A_{0}, l a t}^{(0)}$ but with $O\left(\alpha_{s}\right)$ matching for that current. Green pluses include $J_{A_{0}, l a t}^{(1)}$ in a matching through $O\left(\alpha_{s}\right)$ and purple open squares include the full matching of eqs 1 and 5.

Using matrix elements from [1] we can compare the determination of $B$ meson decay constants from $A_{0}$ and $P$. Figure 3 below shows the comparison for $f_{B}$ as we add successively higher orders in the expansion of the current from eqs 1 and 5. Zeroth order includes only $J^{(0)}$ at tree-level, $O\left(\alpha_{s}\right)$ includes the appropriate $z_{0}$ etc., as described in the figure caption. We take the experimental $B$ meson mass for $m_{B}$ [7], needed in taking the ratio plotted (from eqs. 3 and 4). We set the mass ratio $m_{l} / m_{b}$ to zero since it is of negligible size compared to our error bars.

The dashed black lines in Figure 3 give the relative uncertainty in $f_{B}$ quoted in [1]. We see that, as we improve the approximation to the continuum current, the ratio of the two determinations of the decay constant gets closer to 1 . The best result, using the full $\alpha_{s} \Lambda / m_{b}$ matching that we have, is within the quoted uncertainty.

From our results at multiple values of the lattice spacing and sea quark masses we can determine physical values for $f_{B}$ and $f_{B_{s}}$ using the pseudo scalar density. The ratio also shows good agreement with the results from [1].

A similar analysis for the temporal vector and scalar currents gives a similar plot to that of Figure 3 for the comparison of the scalar form factor for $B \rightarrow \pi \ell v$ decay at zero recoil using matrix elements from [8]. In Figure 4 we show the comparison for the ratio $R_{B \pi}$, defined as:

$$
R_{B \pi}=\frac{f_{0}\left(q_{\max }^{2}\right) f_{\pi}}{f_{B}}
$$

obtained with the two methods. Again we see good agreement within the quoted uncertainty as successively better approximations to the continuum current are made. 


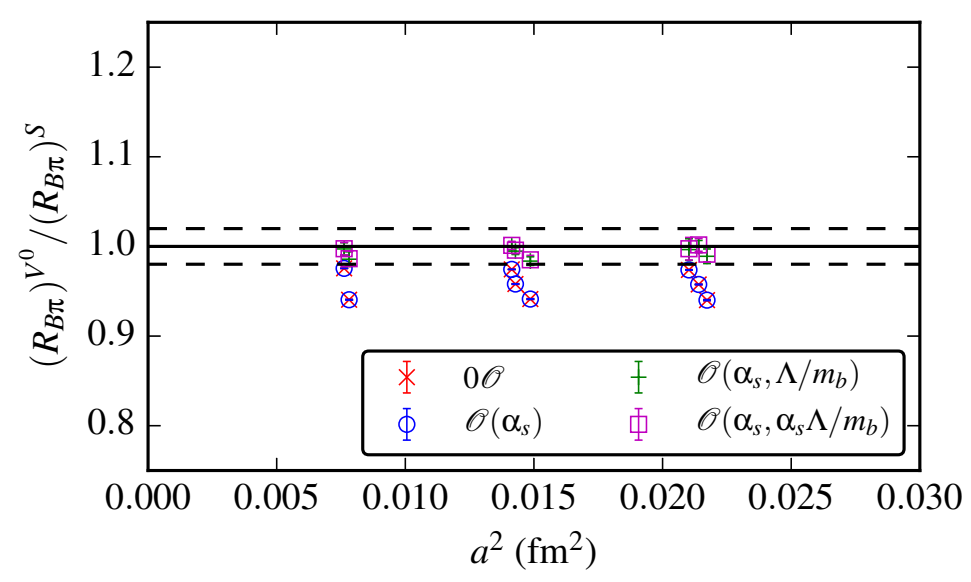

Figure 4. The ratio $R_{B \pi}$ (defined as the ratio of the scalar form factor at zero recoil to the decay constant ratio $f_{B} / f_{\pi}$ (eq. 6)) obtained using the temporal vector current to that obtained using the scalar density, plotted against the square of the lattice spacing. Results are obtained using lattice QCD calculations for NRQCD $b$ quarks and HISQ light quarks and plotted for successively more accurate non-relativistic expansions of the continuum current operator. We use a variety of ensembles with different lattice spacing and sea quark masses (giving multiple results at each value of the lattice spacing). Symbols are as for Figure 3. Note that in this case the zeroth order and $O\left(\alpha_{s}\right)$ results agree with each other because of cancellations in forming $R_{B \pi}$.

\section{Conclusions}

In Figure 5 we give a summary plot of lattice QCD determinations of $f_{B}, f_{B_{s}}$ and their ratio. Here $f_{B}$ is defined as the decay constant for a $B$ meson corresponding to a light quark with the $u / d$ average mass. The HPQCD results are: red open squares for the $A_{0}$ current, red open circles for the $P$ current, and a blue diamond for the average (allowing for correlations through the input data).

In future this analysis will enable us to determine both the vector and scalar form factors away from zero recoil for $B \rightarrow \pi \ell v$ decay by combining results from temporal vector and scalar currents. As we show here, both of these currents can be matched to their continuum counterparts including the effect of the sub-leading $J^{(2)}$ current which appears at $O\left(\alpha_{s}\right)$ in the expansion (eqs 1 and 5). This current is important because it includes a derivative on the light quark field and so its matrix element grows in size, relative to that of $J^{(0)}$, as the $\pi$ meson in the final state has increasing momentum away from zero recoil. A matching that includes this current then has reduced systematic uncertainties in the shape of the form factor away from zero recoil.

Acknowledgements: We are grateful to the MILC collaboration for the use of their configurations and to R. Dowdall and B. Colquhoun for determination of the matrix elements needed in this calculation. Computing was done on the Darwin supercomputer at the University of Cambridge as part of STFC's DiRAC facility. We are grateful to the Darwin support staff for assistance. Funding for this work came from the Royal Society, the Wolfson Foundation and STFC. Fermilab is operated by Fermi Research Alliance, LLC, under contract number DE-AC02-07CH11359 with the U.S. Department of Energy. CJM is supported in part by the U.S. Department of Energy under grant number DE-FG02-00ER41132. 


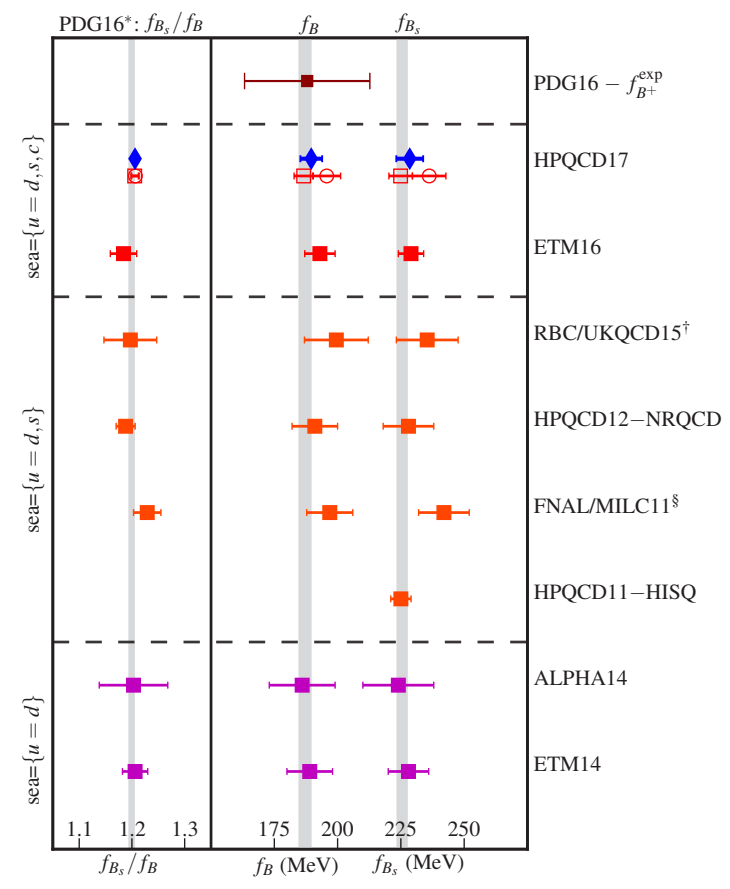

Figure 5. A summary of lattice QCD calculations for $f_{B}, f_{B_{s}}$ and their ratio. The new results reported here are the those for the pseudoscalar current given by the red open circles, showing good agreement with earlier results (given by red open squares) using the temporal axial current [1]. The average of these results is given by blue diamonds. Other results in this summary are taken from [9-15] and use a variety of different quark formalisms for heavy and light quarks as well as working with gluon field configurations that include different numbers of flavours of sea quarks. The results for $f_{B}$ correspond to those for a light valence quark of mass equal to the average of $u$ and $d$ quark masses except for 'RBC/UKQCD15' which correspond to the neutral $b \bar{d}$ meson and 'FNAL/MILC11' which correspond to the charged $b \bar{u}$ meson. The experimental result for the charged $B$ meson is an average from the Particle Data Group [7], as are the grey bands. 


\section{References}

[1] R. Dowdall, C. Davies, R. Horgan, C. Monahan, J. Shigemitsu (HPQCD Collaboration), Phys.Rev.Lett. 110, 222003 (2013), 1302 . 2644

[2] R.J. Dowdall et al. (HPQCD), Phys. Rev. D85, 054509 (2012), 1110. 6887

[3] C. Monahan, J. Shigemitsu, R. Horgan, Phys. Rev. D87, 034017 (2013), 1211.6966

[4] A. Bazavov et al. (MILC Collaboration), Phys.Rev. D87, 054505 (2013), 1212 . 4768

[5] A. Bazavov et al. (Fermilab Lattice, MILC), Phys. Rev. D90, 074509 (2014), 1407.3772

[6] R. Dowdall, C. Davies, G. Lepage, C. McNeile (HPQCD Collaboration), Phys.Rev. D88, 074504 (2013), 1303.1670

[7] C. Patrignani et al. (Particle Data Group), Chin. Phys. C40, 100001 (2016)

[8] B. Colquhoun, R.J. Dowdall, J. Koponen, C.T.H. Davies, G.P. Lepage, Phys. Rev. D93, 034502 (2016), 1510.07446

[9] A. Bussone et al. (ETM), Phys. Rev. D93, 114505 (2016), 1603.04306

[10] N.H. Christ, J.M. Flynn, T. Izubuchi, T. Kawanai, C. Lehner, A. Soni, R.S. Van de Water, O. Witzel, Phys. Rev. D91, 054502 (2015), 1404 . 4670

[11] H. Na, C.J. Monahan, C.T.H. Davies, R. Horgan, G.P. Lepage, J. Shigemitsu, Phys. Rev. D86, 034506 (2012), 1202.4914

[12] A. Bazavov et al. (Fermilab Lattice, MILC), Phys. Rev. D85, 114506 (2012), 1112 . 3051

[13] C. McNeile, C.T.H. Davies, E. Follana, K. Hornbostel, G.P. Lepage, Phys. Rev. D85, 031503 (2012), 1110.4510

[14] F. Bernardoni et al. (ALPHA), Phys. Lett. B735, 349 (2014), 1404.3590

[15] N. Carrasco et al. (ETM), JHEP 03, 016 (2014), 1308. 1851 